EPJ Web of Conferences 59, 05004 (2013)

DOI: $10.1051 /$ epjconf/20135905004

(C) Owned by the authors, published by EDP Sciences, 2013

\title{
Effects of hydrodynamics on Stimulated Brillouin Scattering in multiple plasma interaction
}

\author{
V. Yahia ${ }^{1, a}$, S. Depierreux², C. Goyon ${ }^{2}$, G. Loisel ${ }^{1}$, N.G. Borisenko ${ }^{3}$, \\ P.E. Masson-Laborde ${ }^{2}$, A. Orekhov ${ }^{3}$, T. Rienecker ${ }^{4}$, O. Rosmej ${ }^{4}$ \\ and C. Labaune ${ }^{1}$ \\ 1 LULI, Ecole Polytechnique, CNRS, CEA, UMPC, 91128 Palaiseau Cedex, France \\ 2 CEA, DAM, DIF, 91297 Arpajon, France \\ 3 P.N. Lebedev Physical Institute, Russian Academy of Science, Moscow, Russia \\ ${ }^{4}$ GSI Helmhotzzentrum für Schwerionenforschung, 64291 Darmstadt, Germany
}

\begin{abstract}
In this paper, an experiment carried out on LULI2000 facility is presented. It was designed to investigate how two successive plasmas interact through hydrodynamic coupling and electromagnetic radiations. Contributions of both effects have been successfully identified and the effects of hydrodynamic coupling on Stimulated Brillouin Scattering has been observed.
\end{abstract}

\section{INTRODUCTION}

Laser-Plasma Interaction physics (LPI) is one of the major concerns for megajoule class laser facilities, aiming at achieving laser-driven fusion [1,2]. Huge progress has been made in understanding laser propagation and energy deposition inside the cavity, and parametric instabilities are now under a better control. Nonetheless, in spite of the progress that has been made in modeling, current codes are not able to reproduce the process for its whole duration and with its whole complexity. This complexity arises from the large number of beams involved, the long duration of pulses and the huge dimension of the plasma compared with the typic scale of the studied phenomena. The latter can be strongly affected by beam bending or beam crossing, as well as electromagnetic seeding [3, 4]. These predictability limitiations have been highlighted in very recent National Ignition Facility (NIF) experiments [5, 6]. Backscattered light measurements have shown that cross-beam energy transfer $[6,7]$ noticeably modify irradiation symmetry and reflectivity levels with regard to codes predictions. Understanding physical processes in such a complex situation is difficult. In this context, we designed an experiment devoted to investigate related LPI in a more simple configuration. Particularly, we investigated the case of one beam propagating through a succession of two plasmas, thus reproducing what happens to an isolated beam propagating through a hohlraum. Before reaching the wall of the cavity, the laser beam propagates through a low density gas that prevents the walls from expanding too much. This creates a long and hot undercritical plasma. When reaching the wall, the laser then creates another plasma that expands against the first one. In this situation, LPI is strongly affected by hydrodynamic coupling of the plasmas. Moreover, scattered light from one of the plasmas can be reamplified in the other one, acting as an electromagnetic seed for instabilities [8]. Combination of these two phenomena may lead to higher reflectivities.

\footnotetext{
ae-mail: vincent. yahia@polytechnique.edu
}

This is an Open Access article distributed under the terms of the Creative Commons Attribution License 2.0, which permits unrestricted use, distribution, and reproduction in any medium, provided the original work is properly cited. 


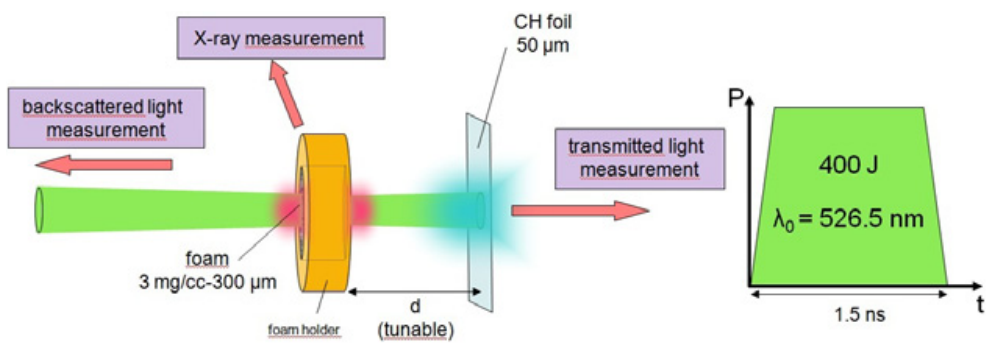

Figure 1. Experimental scheme designed to investigate multiple plasmas interaction.

\section{LULI2000 EXPERIMENT}

The experiment depicted in this paper has been performed using one of the nanosecond beams of the LULI2000 laser facility to its second harmonic $(526.5 \mathrm{~nm})$. We chose targets and irradiation geometry so that our conditions are the most representative as possible of what occurs inside cavities. For this purpose, we used underdense foam targets which provide millimetric scale homogeneous plasma and reach a high electron temperature [9]. We also made a point at separating the major effects that happen together in fusion experiments. For this reason, we have developed a versatile target system, that allowed us to irradiate two targets at the same time, with a tunable distance d between them. In this paper, we report results obtained using a combination of a $3 \mathrm{mg} / \mathrm{cc}(0.24 \mathrm{nc}$ at $526.5 \mathrm{~nm}) 300 \mu \mathrm{m}$ long foam followed by a $50 \mu \mathrm{m}$ thickness $\mathrm{CH}$ foil. Both targets were irradiated using a $1.5 \mathrm{~ns} 400 \mathrm{~J}$ square pulse both for plasma creation and interaction. Random Phase Plate (RPP) was used on the beam, providing a well-defined laser intensity distribution in the focal spot, reaching a maximum average value of $5 \times 10^{14} \mathrm{~W} / \mathrm{cm}^{2}$. Considering the narrow aperture of the beams (f/8), the resulting focal depth is approximately $4.5 \mathrm{~mm}$. Thus, the intensity can be considered to be the same on both parts of the target, for all the investigated distances. The main diagnostics used in this experiment were backscattered light measurements and transmitted light measurements. Stimulated Raman Scattering (SRS) and Stimulated Brillouin Scattering in the backward direction were spectrally and temporally resolved using spectrostreak combinations and backscattered power was measured using photodiodes. The transmitted light has been diagnosed by power measurement as well as a 1D time-resolved imaging of the rear side of the plasma. At last, we implemented X-ray Von Hamos spectrometers, X-ray pinholes and X-ray diodes for temperature measurement. For the compound target, three distances have been investigated: $0 \mathrm{~mm}$, $0.6 \mathrm{~mm}, 1.2 \mathrm{~mm}$. Experimental scheme is depicted in Fig. 1 .

\section{EXPERIMENTAL RESULTS}

We report in this section results on hydrodynamic coupling of the two plasmas. The parameter $2 c_{s} \tau$ gives the order of magnitude for the minimal distance to put between the targets, in order to be free from hydrodynamic coupling of the plasmas during the pluse duration $\tau$. In this expression, $c_{s}$ is the sound speed given by $c_{s}=\sqrt{\frac{Z T_{e}+3 T_{i}}{A m_{p} c^{2}}}$, where $\mathrm{Z}$ is the averaged degree of ionization, $T_{i}$ is the ion temperature, $\mathrm{A}$ is the averaged mass number and $m_{p}$ is the proton mass. According to the measured $\mathrm{X}$-ray spectra, electron temperature for the foam is $1.0 \pm 0.2 \mathrm{keV}$ in good agreement with our numerical simulations. The latter give us $T_{i}=0.25 \mathrm{keV}$. We then find that $2 c_{s} \tau=0.72 \mathrm{~mm}$. Following this calculation, compound targets with $\mathrm{d}=1.2 \mathrm{~mm}$ should be free from hydrodynamic coupling, contrary to compound targets with $\mathrm{d}=0.6 \mathrm{~mm}$ and $0 \mathrm{~mm}$.

Comparison between SBS time-resolved spectra for different distances between the targets shows strong modifications in SBS behaviour. In Fig. 2, we compare SBS spectra of the compound target for $\mathrm{d}=1.2 \mathrm{~mm}, \mathrm{~d}=0.6 \mathrm{~mm}$ and $\mathrm{d}=0 \mathrm{~mm}$. The transmitted light profile as a function of time, that 

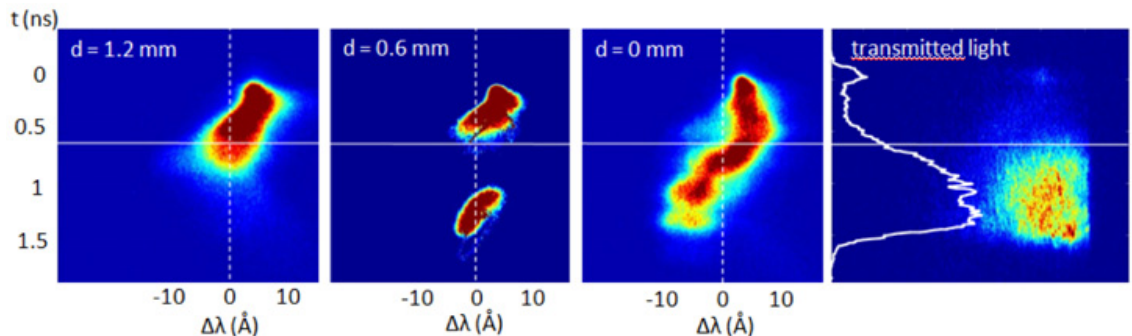

Figure 2. Time resolved SBS spectra for $\mathrm{d}=1.2-0.6-0 \mathrm{~mm}$. On the right, time resolved transmitted light.

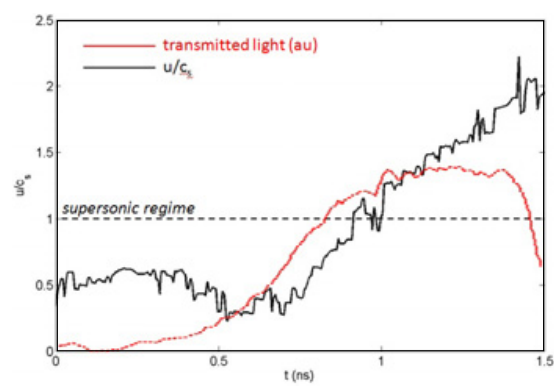

Figure 3. Plasma flow velocity where the SBS occurs, infered from the spectral shift in the case $\mathrm{d}=0 \mathrm{~mm}$.

was obtained from our transmission diagnostic for a shot on foam alone, has also been plotted. One can see that after the foam begins to transmit ( $t=0.7 \mathrm{~ns})$, the spectrum for $\mathrm{d}=0 \mathrm{~mm}$ is strongly and rapidely blueshifted to more than 6 (central wavelength at the end of the spectrum), compared to the one for $\mathrm{d}=1.2 \mathrm{~mm}$ that hardly shifts under the laser wavelength $(\Delta \lambda=0)$. For $\mathrm{d}=0.6 \mathrm{~mm}$, two distinct components are seen. The first component shifts toward short wavelengths with time. As the foam becomes transparent, a redshifted component appears.

The shift in SBS spectra is related to the plasma hydrodynamic parameters, according to the relation $\Delta \omega=2 \omega_{0} \frac{c_{s}-u(z)}{c} \sqrt{1-\frac{n_{e}}{n_{c}}}$, where $\omega_{0}$ is the laser frequency, $c$ is the light velocity, $n_{e}$ is the electron density, $n_{c}$ is the critical density and $\mathrm{u}(\mathrm{z})$ is the expansion velocity profile along the laser axis. The blueshift can then be related to a change in the plasma flow, induced by the presence of the foil plasma very close to the foam.

Using time evolution of the electron temperature given by hydro-simulations, one can extract from the shift formula the time evolution of plasma flow velocity, which is plotted in Fig. 3 for the case $\mathrm{d}=0 \mathrm{~mm}$. The time resolved transmitted light for the foam alone has also been plotted on the same graph. At the begining of the laser pulse, the transmitted light level remains low. The transmitted light comes from the ionization front into the foam that scatters laser light forward into the diagnostic. In the plasma behind the ionization front, SBS takes place as can be seen in the spectra. The associated velocity of plasma flow is found to be subsonic and approximately constant over this period. Then, the foam is fully ionized, leading to an undercritical plasma transparent to the laser light. This is visible on the transmitted light level that rapidely increases. The foil behind the foam is then irradiated, leading to a hydrodynamic interaction between the two plasmas. This interaction strongly affects the plasma flow velocity infered from SBS spectrum that rapidely increases and enters the supersonic regime.

In Fig. 4, time integrated reflectivity has been plotted as a function of the distance between the targets, as well as the power reflectivity. It can be seen that integrated reflectivity is constant at $18 \%$ for $\mathrm{d}=0 \mathrm{~mm}$ and $\mathrm{d}=0.6 \mathrm{~mm}$ before decreasing to $12 \%$ for $\mathrm{d}=1.2 \mathrm{~mm}$. Looking at the power 

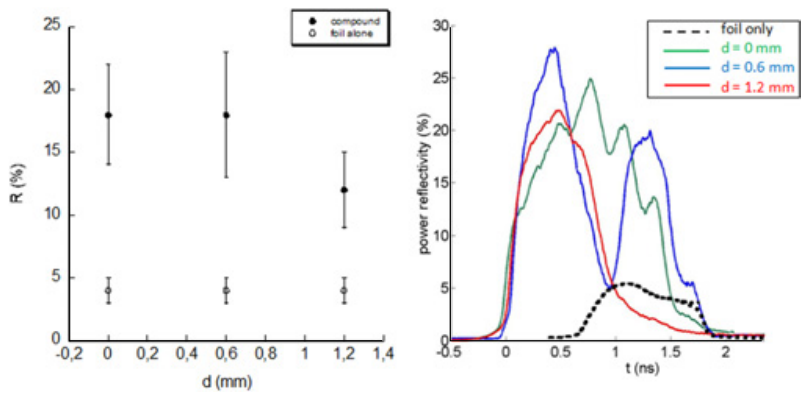

Figure 4. Time integrated reflectivities (left) for compound targets (dots) and foil alone (circles). Power reflectivities as a function of $\mathrm{d}$ (right).

reflectivities, one can see that for times earlier than $0.7 \mathrm{~ns}$ there is no significant difference between the three configurations. After that time, when the foil is ionized, power reflectivity for $\mathrm{d}=0 \mathrm{~mm}$ remains at a high value (around 20\%) whereas it drops dramatically for $\mathrm{d}=1.2 \mathrm{~mm}$. For $\mathrm{d}=0.6 \mathrm{~mm}$, a second burst of reflectivity, peaking at $20 \%$, appears at $\mathrm{t}=1 \mathrm{~ns}$. As this effect is a function of the distance between the targets, it is clearly related to hydrodynamics.

\section{CONCLUSION}

By irradiating low-density foam, we created a long (hundreds of microns) hot (Te $\approx 1 \mathrm{keV}$ ) plasma, relevant for fusion experiments. We designed tunable compound targets to mimic hohraum two plasma configuration. It is clear from reflectivities and spectral shifts that SBS is strongly affected by the hydrodynamic coupling of the two plasmas. When this kind of coupling happens, we observed that the SBS duration is increased as well as the reflectivity levels. Power reflectivities as high as $20 \%$ have been observed for almost the whole pluse duration for hydrodynamicaly coupled plasmas. In the region where SBS occurs, strong modification of the plasma flow velocity has been observed when the coupling occurs. Simulations of these complex targets must be performed in order to fully understand the hydrodynamics of the system, thus providing a complete explanation of the effect on reflectivity levels. Results concerning electromagnetic coupling will be displayed in a subsequent publication.

\section{References}

[1] J. H. Nuckolls et al., Nature 239, 139 (1972)

[2] J. D. Lindl et al., Phys. Plasmas 11, 339 (2004)

[3] H. A. Baldis et al., Phys. Rev. Lett. 77, 2957 (1996)

[4] W. Seka et al., Phys. Rev. Lett. 89, 175002 (2002)

[5] D. E. Hinkel et al., Phys. Plasmas 18, 056312 (2011)

[6] P. Michel et al., Phys. Rev. Lett. 102, 025004 (2009)

[7] R. K. Kirkwood et al., Phys. Plasmas 76, 2065 (1996)

[8] J. C. Fernández et al., Phys. Rev. Lett. 81, 2252 (1998)

[9] K. A. Tanaka et al., Phys. Fluids 27, 2910 (1985) 